\title{
Los derechos de la niñez en México: Una agenda más que incumplida
}

\author{
The rights of children in México: An agenda rather than unfulfilled \\ Héctor Sánchez Pérez ${ }^{1}$, Anaximandro Gómez Velasco²
}

\section{RESUMEN}

Objetivo: Analizar la situación de desprotección de la niñez mexicana, con base a diversos indicadores de salud y de acceso a los servicios de salud. Materiales y métodos: se analizaron indicadores oficiales de salud (morbilidad, mortalidad, desnutrición y muerte materna, entre otros) y de acceso a los servicios de salud (vacunación). Resultados: diversas enfermedades infecciosas, como la neumonía e influenza, y las enfermedades intestinales -gran parte de ellas, evitables-, continúan estando entre las primeras causas de muerte en niños de 0-4 años de edad. En niños de 1-5 años, la tasa de mortalidad es muy alta en regiones con muy alta marginación socioeconómica, alcanzando hasta 21.3 por cada 100,000 niños. Las zonas rurales tienen un bajo porcentaje de seguridad alimentaria, alcanzado solo el 19.2\% de los hogares. La razón de mortalidad materna es muy alta en regiones con muy alta marginación socioeconómica, alcanzando valores de hasta 65.3 por cada 100,000 nacidos vivos. El porcentaje de vacunación en menores de 1 año solo alcanza el $49.8 \%$. Conclusión: los indicadores de salud analizados, evidencian que las y los niños mexicanos sufren de desprotección por parte del Estado mexicano, en uno de sus derechos fundamentales, la salud. Es necesario re-orientar las políticas de protección a la niñez mexicana con el fin de que verdaderamente se garanticen y cumplan sus derechos humanos.

Palabras Clave: Derechos del niño, derecho a la salud, México.

\begin{abstract}
Objective: To analyze the lack of protection of Mexican children based on several health indicators, as well as access to health services. Material and methods: official health indicators (morbidity, mortality, malnutrition and maternal mortality, among others) and access to health services (vaccination) were analyzed. Results: various infectious diseases, such as pneumonia and influenza, as well as intestinal diseases -most of them preventable-, still continue in the first places to cause death in children 0-4 years old. In children aged 1-5 years, the mortality rate is very high in marginalized regions, reaching up to 21.3 per 100,000 children. Rural areas have a low percentage of food security reached only $19.2 \%$ of houses. The maternal mortality ratio is very high in regions with very high poverty reaching values of up to 65.3 per 100,000 live births. The vaccination rate for children under one year only reached $49.8 \%$. Conclusion: The health indicators analyzed show that Mexican children lack protection by the Mexican State in one of their fundamental rights, health. It is necessary to change the policies to protect Mexican children in order to truly guarantee and meet their human rights.
\end{abstract}

Key words: Children right, right to health, Mexico.

\footnotetext{
1 Ph.D. Colegio de la Frontera Sur (ECOSUR), Unidad San Cristóbal de las Casas. México.

${ }^{2}$ Ph.D. Nodo GRAAL (Grupos de Investigación para América y África Latinas) - ECOSUR
} 


\section{INTRODUCCIÓN}

Las y los niños constituyen uno de los grupos poblacionales más protegidos por las leyes internacionales y de cada país en particular. Para tener una idea de ello, baste citar algunos ordenamientos que, de una u otra forma se relacionan con su protección y bienestar, y de los que se supone los Estados deben garantizar su cumplimiento (1). Por ejemplo, en el ámbito internacional, se tiene la Convención Internacional sobre los Derechos de la Niñez, el Protocolo sobre la Participación de Menores en Conflictos Armados, el Protocolo Facultativo de la Convención sobre los Derechos del Niño relativo a la venta de niños, la prostitución infantil y la utilización de niños en la pornografía, la Declaración Universal de los Derechos Humanos, la Convención Americana sobre Derechos Humanos (Pacto de San José), el Pacto Internacional de Derechos Económicos, Sociales y Culturales (PIDESC) y la Convención sobre la Eliminación de Todas las formas de Discriminación contra la Mujer (Convención de la Mujer), entre otros (1). En México, particularmente la Constitución Política de los Estados Unidos Mexicanos (2) y la Ley Federal para la Protección de los Derechos de Niñas, Niños y Adolescentes (3) protegen y promueven los derechos fundamentales de este grupo poblacional.

Sin embargo, ni los internacionales (considerados al mismo nivel que la Constitución Política mexicana), ni los del propio país, se cumplen satisfactoriamente. Para dar cuenta de ello, el presente trabajo ofrece un breve análisis de indicadores de salud y de acceso a los servicios de salud, que dan cuenta del incumplimiento de los derechos del niño en México.

\section{MATERIAL Y MÉTODOS}

Revisión documental. Se revisaron las estadísticas oficiales de indicadores de salud (morbilidad, mortalidad, desnutrición, entre otros) y de acceso a los servicios de salud (vacunación) de la niñez en México.

\section{RESULTADOS}

Salud y acceso a los servicios de salud: De acuerdo al Programa Sectorial de Salud 2013-2018 del gobierno de la república, en el año 2011, los accidentes, la neumonía e influenza y las enfermedades infecciosas intestinales se ubicaron en las primeras cinco causas de muerte en las y los niños de 0 a 4 años de edad (4). En las y los de 5 a
11 años, los accidentes y las agresiones (homicidios) estuvieron entre las primeras cinco causas de muerte y, entre las y los de 12-19 años de edad, tres de las cinco principales causas de muerte fueron las ya citadas en el grupo de 5-11, pero también las lesiones autoinflingidas intencionalmente (suicidios) (4).

Para ese mismo año, según cifras oficiales, la tasa de mortalidad infantil (TMI -cociente que divide el número de niños nacidos vivos muertos antes de cumplir el año de edad) en los municipios de muy bajo grado de marginación fue de 12,3 por 100000 nacidos vivos, en tanto que para los municipios de muy alto grado de marginación fue de 15.4 en igual denominador (4). Por su parte, en los menores de 1-5 años de edad, dichas cifras fueron, en el orden citado, de 14,6 y 21,3 por 100,000 del grupo. En este sentido, la meta establecida de acuerdo a los Objetivos del Milenio (ODM) era de 10,8 y de 13,7 para los menores de un año y para los de 1-5 años, respectivamente (5).

En lo que respecta a la prevalencia de desnutrición, según la Encuesta Nacional de Salud y Nutrición de 2012 (ENSANUT), mientras a nivel nacional la baja talla para la edad en menores de cinco años fue de $13.6 \%$, en los niños y niñas de la zona norte fue de $8.9 \%$, pero en los estados del sur (donde se encuentran Chiapas, Oaxaca y Guerrero, tres de las entidades más pobres del país) fue de $19.2 \%$, es decir, 2.2 veces mayor que sus pares de la zona norte de México (6). Peor aún, en lo que se refiere a seguridad alimentaria, en el área urbana solo el 33\% de los hogares tiene acceso a alimentos, mientras en el área rural apenas lo tiene el 19.2\% (6). En ambos ámbitos, alrededor de uno de cada diez hogares está considerado por las propias autoridades del sector salud en México con “inseguridad alimentaria severa” (6).

A pesar de dichas cifras carenciales, al llegar a la adolescencia, y según datos de la ENSANUT 2012, el 35\% de las y los adolescentes de entre 12 y 19 años presenta sobrepeso u obesidad (uno de cada cinco tiene sobrepeso y uno de cada diez presenta obesidad) (6).

Un indicador especialmente sensible a las condiciones en las que se desarrollará un/a niño/a, lo constituye la muerte materna, debido a la importancia de los vínculos psico-emocionales y cuidados, alimentación, entre otros aspectos, que le puede -o no- proporcionar la madre al niño/a. En 2011, mientras la razón de mortalidad materna (RMM) para el país en su conjunto fue de 43 muertes maternas por cada 100,000 nacidos vivos, 
en los estados considerados de muy baja marginación (Distrito Federal, Nuevo León, Coahuila y Baja California) fue de 37 , en los de muy alto grado de marginación (Oaxaca, Chiapas y Guerrero), fue de 65,3 en el mismo denominador $(4,5)$.

En cuanto a vacunación, según la ENSANUT 2012, poco menos de la mitad de los menores de un año (49,8\%) tiene completo su esquema de vacunación y, en los de un año de edad, dicha cifra es del $60.7 \%$ (6).

Por otra parte, de acuerdo al Programa Sectorial de Salud 2013-2018, no se ha podido reducir a cero el número de casos de transmisión vertical del $\mathrm{VIH}$, a pesar de que ya existe acceso al tratamiento antirretroviral gratuito, y, en cuanto a cesáreas, éstas representan el 38.5\% de los nacimientos en el sector público (que van desde $23 \%$ de los partos atendidos en el Programa IMSS-Progresa (antes IMSS-Oportunidades), hasta $67 \%$ en el Instituto de Servicios y Seguridad Social para los Trabajadores del Estado (ISSSTE), cuando la recomendación internacional, debe ser menor al 15\% (4).

Algunos indicadores que reflejan la situación de los niños y niñas en el país, con respecto a otros países de la Organización para la Cooperación y Desarrollo Económico (OCDE) a la que México pertenece, (Tabla 1).

Tabla 1. México en la OCDE. Indicadores de salud 2011 (o el año más cercano).

\begin{tabular}{|c|c|c|c|}
\hline Indicador & México & OCDE & Observaciones \\
\hline Esperanza de vida al nacer (años) & & & Fuente: a,b \\
\hline Ambos sexos & & & \\
\hline Hombres & 74.2 & 80.1 & \\
\hline Mujeres & 77.2 & 82.8 & \\
\hline & 71.2 & 77.3 & \\
\hline $\begin{array}{l}\text { Tasa de Mortalidad Infantil (TMI) } \\
\text { (por 1,000 nacidos vivos) }\end{array}$ & 13.6 & 4.1 & $\begin{array}{l}{ }^{\mathrm{c}} \text { Los de menor MI son Islandia } 1.6 \text { y } \\
\text { Suiza, 2.1; México es el } 5^{\circ} \text { lugar de } \\
\text { mayor MI, por debajo de Brasil (13.9), } \\
\text { Indonesia (24.8), Sudáfrica (34.6) e India } \\
\text { (47.2) (3) }\end{array}$ \\
\hline $\begin{array}{l}\text { TM neonatal (por 1,000 nacidos } \\
\text { vivos) }\end{array}$ & 8.6 & 2.8 & Fuente: a,c \\
\hline \multirow{2}{*}{$\begin{array}{l}\text { Razón de Mortalidad Materna (por } \\
100,000 \text { nacidos vivos) }\end{array}$} & & & Fuente: a,b \\
\hline & 43.0 & 7.3 & \\
\hline \% Bajo peso al nacer & 8.6 & 6.8 & $\begin{array}{l}\text { c Islandia (3.2) y Finlandia (4.1) son los } \\
\text { de menor \% de BPN. Sudáfrica (13.2) e } \\
\text { India (27.6) son los de mayor \%. } \\
\text { México es el 7o más alto. }\end{array}$ \\
\hline $\begin{array}{l}\text { \% sobrepeso en niños, incluyendo } \\
\text { obesidad (2010) }\end{array}$ & 30.0 & 21.0 & $\begin{array}{l}{ }^{\mathrm{C}} \text { Grecia e Italia son los de mayor } \\
\text { prevalencia (con } 40 \% \\
\text { aproximadamente). México es el } 6^{\circ} \text { más } \\
\text { alto; los de menor prevalencia son } \\
\text { Sudáfrica }(10 \%) \text { e Indonesia }(8 \%) \text {. }\end{array}$ \\
\hline
\end{tabular}

a INEGI (2013) Los objetivos de desarrollo del milenio en México. Informe de Avances 2013, Gobierno de la República de México, México, D.F.

b SSA (2014) Programa Sectorial de Salud 2013-2018, Plan Nacional de Desarrollo, Gobierno de la República de México, México, D.F.

c OECD (2013), Health at a Glance 2013: OECD Indicators, OECD Publishing. Disponible en: http://dx.doi.org/10.1787/health_glance-2013. 
Algunos indicadores de gasto en el sector salud 2011, en México, así como el gasto en salud per cápita de 977 USD, gasto en salud como \% del producto interno bruto (PIB), es de 6,2 ; gasto administrativo en el sector salud es del $10 \%$; las unidades de imágenes de resonancia magnética (por millón de habitantes) es de 2,1; número de camas hospitalarias por 1,000 habitantes, es de 1,5; médicos por 1,000 habitantes* es de 2,2; enfermeras por 1,000 habitantes, es de 2,7. (Tabla 2$,$) .$

Tabla 2. México en la OCDE. Indicadores de gasto en el sector salud 2011 (o el año más cercano).

\begin{tabular}{|c|c|c|c|}
\hline Indicador & México & OCDE & Observaciones \\
\hline $\begin{array}{l}\text { Gasto en salud per cápita (en } \\
\text { USD, PPPs - paridad de poder } \\
\text { adquisitivo) }\end{array}$ & $\begin{array}{l}977 \text { (año } \\
2010)\end{array}$ & 3,332 & $\begin{array}{l}{ }^{\mathrm{a}} \text { Los más altos son: EUA, 8,508 y Noruega } \\
\text { con 5,669. México es el } 6^{\circ} \text { más bajo. El de } \\
\text { menor gasto en salud es Indonesia, con } 127 .\end{array}$ \\
\hline $\begin{array}{l}\text { Gasto en salud como \% del } \\
\text { Producto Interno Bruto (PIB) }\end{array}$ & 6.2 & 9.3 & $\begin{array}{l}{ }^{\mathrm{a}} \text { Los de mayor gasto son EUA, } 17.7 \text { y } \\
\text { Holanda, } 11.9 \text {; México, es el } 6^{\circ} \text { más bajo. El } \\
\text { de menor gasto, Indonesia, } 2.7 \text {. }\end{array}$ \\
\hline $\begin{array}{l}\text { Gasto administrativo en el } \\
\text { sector salud (\% del gasto en } \\
\text { total, 2012) }\end{array}$ & 10 & 4.0 & $\begin{array}{l}\text { b Si sólo se considera al sector público, el } \\
\text { gasto administrativo asciende a casi el 17\%. }\end{array}$ \\
\hline $\begin{array}{l}\text { Unidades de imágenes de } \\
\text { resonancia magnética (por } \\
\text { millón de habitantes) }\end{array}$ & 2.1 & 13.3 & $\begin{array}{l}\text { a Japón (46.9) y EUA (31.5) son los de } \\
\text { mayor número. México es el más bajo, } \\
\text { seguido de Israel (2.5) }\end{array}$ \\
\hline $\begin{array}{l}\text { No. de camas hospitalarias por } \\
1,000 \text { habitantes }\end{array}$ & 1.5 & 4.8 & $\begin{array}{l}\text { a,b Los más altos, Japón (13) y Corea (9.2). } \\
\text { México es el 3o más bajo, arriba de } \\
\text { Indonesia e India con menos de } 1.0 \text {. Del total } \\
\text { de camas, } 24 \% \text { son del sector privado, por lo } \\
\text { que el número de camas por 1,000 habitantes } \\
\text { es de } 1.3 \text {. }\end{array}$ \\
\hline $\begin{array}{l}\text { Médicos por } 1,000 \\
\text { habitantes* }\end{array}$ & 2.2 & 3.2 & $\begin{array}{l}{ }^{\mathrm{a}} \text { Los más altos, Grecia } 6.1 \text { y Rusia, } 5.0 \text {; } \\
\text { México el } 10^{\circ} \text { más bajo. El que menos tiene, } \\
\text { Indonesia, } 0.2 \text {. }\end{array}$ \\
\hline $\begin{array}{l}\text { Enfermeras por } 1,000 \\
\text { habitantes }\end{array}$ & 2.7 & 8.7 & $\begin{array}{l}\text { a Los más altos, Suiza con } 45.1 \text { e Islandia } \\
\text { 22.3; el Más bajo México y Turquía con 3.8. }\end{array}$ \\
\hline
\end{tabular}

Nota: * Incluye a los que dan atención y trabajan en el sector salud como directivos, educadores, investigadores, etc

Referencias:

${ }^{a}$ OECD (2013), Health at a Glance 2013: OECD Indicators, OECD Publishing. Disponible en: http://dx.doi.org/10.1787/health_glance-2013-en.

b SSA (2014) Programa Sectorial de Salud 2013-2018, Plan Nacional de Desarrollo, Gobierno de la República de México, México, D.F.

\section{DISCUSIÓN}

Para la dimensión de necesidades, ¿suficientes recursos para la salud?

Ante el panorama anteriormente descrito de necesidades de salud no satisfechas, bien podría esperarse que el Estado mexicano destinara los recursos suficientes para hacer frente a dicho cúmulo de necesidades. Sin embargo, no sucede así. En la tabla 2, se puede apreciar que no se destinan los recursos necesarios y que se tienen indicios que se gastan de manera inadecuada; por ejemplo, el gasto administrativo es notablemente alto si se compara con otros países de la OCDE.
En términos generales, el modelo de atención a la salud prevaleciente en México está enfocado hacia lo curativo, pero sin la suficiente capacidad resolutiva por parte de los servicios. Las políticas en salud no han podido implementarse en su totalidad con un enfoque multidisciplinario, interinstitucional y con una adecuada articulación, tanto en los diversos niveles de gobierno (federal, estatal, municipal y local), como entre los distintos sectores involucrados en la salud de la población (por ejemplo, el educativo, agrario, laboral, entre otros) e, inclusive, dentro del propio sector salud, presenta en muchas de sus áreas, una marcada fragmentación, descoordinación e insuficiente 
cobertura y calidad de los sistemas de información existentes (7).

En este sentido, el propio sector salud reconoce que hay concentración de unidades de diferentes instituciones en las mismas áreas geográficas, dejando sin cobertura a muchas otras y subutilización de algunas por falta de recursos humanos, equipamiento o falta de recursos (4); el equipamiento con frecuencia no es el adecuado o no se tiene y en general, los recursos tecnológicos de apoyo al diagnóstico se ubican en el nivel hospitalario dejando con muy baja capacidad resolutiva al primer nivel de atención (8); y, que la infraestructura es subutilizada por horarios de atención limitados y deterioro de instalaciones por falta de mantenimiento periódico a lo que bien puede agregarse la continua falta de medicamentos en los centros de atención a la salud (9). El mismo gobierno mexicano reconoce que en 2012, sólo el $36 \%$ de las recetas fueron surtidas en el sector de manera completa, lo que ha contribuido a que las farmacias con consultorio hayan crecido en el país de 2010 a 2012 en un $130 \%$ y que este tipo de farmacias atiendan mensualmente al $7 \%$ de la población mediante sus propios consultorios, muchos de los cuales no tienen la adecuada regulación sanitaria y, por lo tanto, existe la posibilidad de prescripción por parte del médico, y la adquisición por parte del paciente, de medicamentos innecesarios (4).

Todo lo anterior repercute en una notable desigualdad en los beneficios al acceso a la salud, un derecho fudamental de todo ser humano, lo cual desfavorece a todos los sectores poblacionales, pero repercute mayormente en la población infantil.

Concluyendo, indicadores desfavorables de salud (vacunación, seguridad alimentaria, tasa de mortalidad infantil, neonatal y materna, entre otros), reflejan la desprotección que sufren los niños y niñas mexicanos. Dado que la salud en los primeros años de vida ( $0-5$ años) es decisiva, por el desarrollo de las capacidades físicas, intelectuales y emotivas de cada niño y niña, las cuales determinan su salud futura, su desempeño escolar y laboral, entre otros aspectos, es necesario y urgente que el Estado mexicano replantee su política de protección a la niñez y realice los cambios a que haya lugar con el fin de que verdaderamente se garanticen y cumplan sus derechos humanos.

\section{REFERENCIAS BIBLIOGRAFICAS}

1. UNICEF. Convención sobre los Derechos del Niño [Internet]. 2015. Available from: http://www.unicef.org/spanish/crc/index_protoc ols.html.

2. Cámara de Diputados, H. Congreso de la Unión,LXII Legislatura. Constitución Política de los Estados Unidos Mexicanos. Constitución publicada en el Diario Oficial de la Federación el 5 de Febrero de 1917 [Internet]. [cited 2014 Oct 4]. Available from: http://www.diputados.gob.mx/LeyesBiblio/htm/ 1.htm

3. Gobierno de los Estados Unidos Mexicanos. Ley General de los Derechos de niñas, niños y adolescentes [Internet]. México, D.F.; 2014 p. 60. Available from: http://www.diputados.gob.mx/LeyesBiblio/pdf/ LGDNNA_041214.pdf

4. SSA. Programa Sectorial de Salud 2013-2018 [Internet]. México, D.F.: Gobierno de la República de México; 2014 p. 138. Available from:

http://www.dged.salud.gob.mx/contenidos/dged/ descargas/index/ps_2013_2018.pdf

5. INEGI. Los objetivos de desarrollo del milenio en México. Informe de Avances 2013 [Internet]. México, D.F.: Gobierno de la República de México; 2013 p. 37. Available from: http://www.undp.org/content/dam/mexico/docs/ Publicaciones/ODM/UNDP-MX-ODMREInformeMex-2013.pdf

6. Gutiérrez J, Rivera-Dommarco J, Shamah-Levy T, Villalpando-Hernández S, Franco A, CuevasNasu L, et al. Encuesta Nacional de Salud y Nutrición 2012. Resultados nacionales. Cuernavaca, Morelos, Mexico: Instituto Nacional de Salud Pública (INSP); 2013 p. 200.

7. Leal G, Sánchez-Pérez H. Seguridad social "Universal" 2013: Plan Nacional de Desarrollo y Sector Asegurador. Universidad Autónoma Metropolitana, El Colegio de la Frontera Sur; 2014.

8. OECD. Health at a Glance 2013 [Internet]. Paris: Organisation for Economic Co-operation and Development; 2013 [cited 2015 Dec 5]. 212 p. Available from: http://www.oecdilibrary.org/content/book/health_glance-2013-en

9. Leal-Fernández G, Sánchez-Pérez HJ, LeónCortés J. La seguridad social universal de Peña Nieto, ¿̇es la reforma que el país necesita? Hacia un consenso mediático. San Cristóbal de las Casas, Chiapas, México: ECOSUR; 2015.

Recibido: $30 / 09 / 2015$

Aceptado: $20 / 12 / 2015$ 